\title{
The Influence of Social Desirability on Self-Reported Sexual Behavior in HIV Survey in Rural Ethiopia
}

\author{
Alexander $\mathrm{Vu}^{1,2^{*}}$, Kiemanh Pham ${ }^{1,2}$, Nhan Tran', Saifuddin Ahmed ${ }^{3}$ \\ ${ }^{1}$ Department of Emergency Medicine, The Johns Hopkins School of Medicine, Baltimore, USA; ${ }^{2}$ Department of International Health, \\ The Johns Hopkins Bloomberg School of Public Health, Baltimore, USA; ${ }^{3}$ Department of Population, Family and Reproductive \\ Health and Department of Biostatistics, The Johns Hopkins Bloomberg School of Public Health, Baltimore, USA. \\ Email: *avu@jhsph.edu
}

Received September $4^{\text {th }}, 2013$; revised October $4^{\text {th }}, 2013$; accepted October $11^{\text {th }}, 2013$

Copyright (C) 2013 Alexander Vu et al. This is an open access article distributed under the Creative Commons Attribution License, which permits unrestricted use, distribution, and reproduction in any medium, provided the original work is properly cited.

\begin{abstract}
Objective: It is in order to examine associations between social desirability (SD) and self-reports of abstinence among youths in rural Ethiopia. Methods: Youths of ages 15 - 24 (114 participants) were administered questionnaire to assess HIV knowledge and primary abstinence and a modified Marlowe-Crowne Social Desirability Scale to assess SD bias. The relationships between SD groups (dichotomized into high and low) and abstinence by various characteristics were assessed by using Fisher's exact p-values. Results: The odds of individuals reporting abstinence were 13.2 times greater in the high SD group compared to the low SD group (p-value 0.002) when adjusted for education, gender, age group, and HIV knowledge. The differences in abstinence between the high and low SD score groups were also examined for selected variables. Conclusions: Individuals who exhibited more SD bias were more likely to report primary abstinence. SD bias should be considered when conducting self-reported surveys to measure the effectiveness of HIV prevention programs.
\end{abstract}

Keywords: Social Desirability Bias; Self-Reported Sexual Behaviors; HIV Surveys

\section{Introduction}

HIV is a significant cause of mortality in sub-Saharan Africa. In 2010, there were an estimated 22.9 million infected people, making up $67 \%$ of HIV infections worldwide [1]. In response to the alarming prevalence of the infection, the President's Emergency Plan for AIDS Relief (PEPFAR) was established to combat the spread of HIV. A significant portion of PEPFAR's efforts was committed to prevent the spread of HIV through the promotion of ABC programs (abstinence, being faithful, condom use). This effort is associated with decreasing the burden of HIV in sub-Saharan Africa where many of PEPFAR's priority countries are located. In Uganda and Zimbabwe, there was an increase in abstinence and a decrease in HIV prevalence [2,3]. Self-reported surveys to measure the sexual behaviors of the beneficiaries of abstinence promotion programs are commonly used to assess the effectiveness of these programs. However, self-reported surveys are prone to the effect of social desirability (SD).

${ }^{*}$ Corresponding author.
SD is the tendency of respondents to reply in a manner which would be viewed positively by their peers or which is consistent with social norms and expectations. SD bias is a particular concern in studies, which measures HIV knowledge, attitudes, and practices (KAP) because of the sensitivity of the subject matter [4]. Questionnaires of sexual behavior have been shown to be susceptible to misreport and underreporting [5]. In Africa in particular, underreporting of sexual activities has been found in reports of sexual behavior in Tanzania and of HIV KAP in rural Uganda [6,7]. Furthermore, it has been shown that demographic factors may be associated with differences in SD. In one study of Malawian teenagers examining different methods of interviews, males responded to questions about sexual behavior differently depending on the interview method while females did not differ in their response [8]. In another study examining reports of non-marital partnerships, there was a different rate in response depending on age [9]. While these studies show that demographic characteristics may be associated with different responses, studies have also shown 
that there may be differences in SD among different ethnic groups. One study, for example, demonstrated a difference in measures of SD between Latinos and nonLatino Caucasians in the United States [10].

To address the issue of misreport in self-reported questionnaires, the Marlow-Crowne Social Desirability Scale (MC-SDS) was developed in 1960 to measure the effect of social desirability [11]. The scale has been validated in the United States, where it was originally developed [12-14]. Subsequently, it has been tested in other high-income countries [15] as well as in low- and middle-income (LMIC) countries [16]. No such studies have been conducted in sub-Saharan Africa. We have tested the MC-SDS and reported on the reliability of the MCSDS in four African countries [17].

The objective of this study is to examine the association between SD bias and the self-reported levels of primary abstinence among unmarried youths, age 15 - 24, in rural Ethiopia. To the best of our knowledge, there is no literature to date which focused on the relationship between SD bias and self-reports of sexual abstinence. We hypothesize that individuals who scored high on MC-SDS were more likely to over-report primary abstinence.

\section{Methods}

\subsection{Formative Research}

During the formative phase of the study, which took place during July 2008, the team consulted with community leaders working in HIV prevention programs, NGOs, and other stakeholders in Ethiopia to assess the feasibility of the study and plan for its implementation; this included pilot-testing of the tool, identifying locations for the study, and defining potential populations for recruitment.

A comprehensive review of the literature was then undertaken to determine the state of knowledge on survey research on HIV in Africa and the effect of SD bias on self-reported surveys. The literature review identified various scales that had been developed to quantify the effects of social desirability bias on self-reported surveys including the scale developed by Edwards [18], the Lie scale from the Eysenck Personality Inventory [19], and the Marlowe Crowne scale. The MC-SDS was chosen because it has been more widely cited in the literature and had been applied in other LMIC settings.

The Marlow Crowne Social Desirability Scale was translated into Amharic by local translators and then back-translated to confirm the language was correct. Under the guidance of researchers from Johns Hopkins, local research assistants pilot-tested the translated questionnaire with a convenience sample to assess the study population's understanding of the translated MC-SDS. Additional edits were made through an iterative process to ensure that the translations captured the intended meaning of the statements.

As the questions were initially designed for use in high-income countries, some statements referenced cultural practices that were not applicable to LMIC. Of the MC-SDS's original 33 statements, five were omitted after the pilot-testing of the translated questionnaire because they referred to situations that were not appropriate for the rural Ethiopian context. The process of modifying the scale and the reliability of the modified MC-SDS was described in our previously published study [17].

\subsection{Sample and Sampling Framework}

This study was part of a larger evaluation of the Mobilizing, Equipping, and Training Approach for Primary Behavior Change in Youth (MET) Program implemented by Samaritan's Purse in rural Ethiopia. The goal of the MET Project was to reduce the incidence of HIV infection through behavior modification, with an emphasis on abstinence and fidelity using a grassroots, community mobilization approach. To take advantage of the infrastructure in place for assessing the program, data collection for this study was undertaken alongside the usual monitoring and evaluation of the MET Project.

Lot quality assurance sampling (LQAS) served as the sampling framework for this study as well as the evaluation of the MET Project. A stratified, three-stage probability-sampling framework was used for recruitment. The catchment area of the study in Ethiopia was divided into six supervisory areas (SA's); in each SA, 19 villages were randomly selected based on a population proportional-to-size method. In each of the 19 villages, a respondent was randomly selected. Using LQAS, a total of 114 individuals were recruited from the entire catchment area.

After the period of pilot-testing and adjustment of the modified MC-SDS, data collectors were trained in social desirability bias, survey methods, and research ethics in November 2008. The trained data collectors obtained verbal consent in private settings; discussed how the collected data were strictly protected; addressed any confidentiality concerns of the participants; and administered in-person the evaluation questionnaires and the modified MC-SDS to each respondent. The Johns Hopkins School of Medicine Institutional Review Board reviewed and approved the study as conforming to the University's policies on ethical research.

\subsection{Measures}

Social desirability was measured using the modified MC-SDS with 28 items. Each of the items asked the respondent to answer with a "true" or "false", with a "true" answer scored as 1 and a "false" answer scored as 0 . 
Thus, the maximum score for the modified MC-SDS was 28, representing the highest degree of social desirability bias while 0 represented the least. The Cronbach's $\alpha$ coefficient was used to estimate the reliability of the instrument for question items that assessed similar constructs [20]. We defined the threshold of acceptable internal consistency or reliability with an $\alpha$ coefficient of 0.70 or greater. The Cronbach's $\alpha$ coefficient for the modified MC-SDS for this study was 0.80 .

HIV knowledge questions used in the study were in accordance to the pre-validated questions and indicators set by the United Nations General Assembly Special Session on HIV/AIDS. Primary abstinence was defined as never having had sex. The participants in this study were men and women between the ages of 15 and 24 who had never been married.

\subsection{Statistical Analyses}

For the purpose of easy interpretation and ease of use among field practitioners, we categorized the SD score into two groups (low and high SD score) and then examined the relationship between abstinence and SD scores by selected characteristics (gender, age group, education level, and HIV knowledge). The determination of SD score cut-off threshold was based on the midpoint separation of the scale. Those who scored below 15 were placed in the low group while those who scored 15 or higher were placed in the high SD score group. Those with no education or only a primary education were grouped into the low education group while those with at least a secondary education were in the high education group. HIV knowledge was defined by correctly answering five questions about how HIV is transmitted. Those who correctly answered all five questions were grouped in the high HIV knowledge group while those who did not answer one of the five questions correctly were grouped into the low HIV knowledge group. The Fisher's exact p-value was used to assess differences between SD score groups. Data was collected and entered into an EpiInfo [21] database. The data were analyzed using Stata/IC, version 11 [22].

\section{Results}

The research team recruited a total of 114 participants. Table 1 shows the background characteristics of the participants. There was nearly an equal number of men (48.2\%) and women (51.8\%). The majority of participants $(80.7 \%)$ were between the ages of 15 - 19, and fewer participants had only a primary education than a secondary education ( $43.0 \%$ and $47.4 \%$, respectively).

Participants were divided into high and low SD score groups. The unadjusted odds of participants in the high SD score group reporting abstinence was 5.21 times greater than those in the low SD score group (p-value 0.002). This relationship was stronger with further adjustment for education, gender, age group, and HIV knowledge (OR = 13.2; p-value 0.002).

Table 2 shows the relationship between SD and abstinence by selected characteristics. The high SD score group had a higher proportion of abstinent respondents than the low SD group regardless of the characteristic. Among males, $86.1 \%$ of high SD score participants reported abstinence compared to $58.3 \%$ of low SD score participants (p-value 0.049). Females reported a significant difference between the two groups with $91.7 \%$ of high SD score individuals reporting abstinence versus 63.6\% of low SD score individuals (p-value 0.033 ).

Almost all (97.3\%) of the individuals in the 15 - 19 age group who had a high SD score reported abstinence while $77.8 \%$ of individuals in the low SD score group were abstinent (p-value 0.013). In the 20 - 24 age group,

Table 1. Background characteristics.

\begin{tabular}{ccc}
\hline Total & $\mathrm{n}=114$ \\
Male & Sex (\%) & $55(48.2)$ \\
Female & & $59(51.8)$ \\
& Age (\%) & \\
$15-19$ & & $22(80.7)$ \\
$20-24$ & & $10(8.3)$ \\
& & $10(8)$ \\
None & & $49(43.0)$ \\
Primary & & $54(47.4)$ \\
\hline
\end{tabular}

Table 2. Percentage of self-reported abstinence between low and high SDS score groups by selected variables.

\begin{tabular}{cccc}
\hline \multicolumn{4}{c}{ Percentage of abstinence (n) } \\
\hline & $\begin{array}{c}\text { Low SD } \\
\text { Score }<\text { 15 }\end{array}$ & $\begin{array}{c}\text { High SD } \\
\text { Score } \geq 15\end{array}$ & $\begin{array}{c}\text { Fisher's Exact } \\
\text { p-value }\end{array}$ \\
\hline Male Gender & $58.3(7 / 12)$ & $86.1(37 / 43)$ & 0.049 \\
Female Gender & $63.6(7 / 11)$ & $91.7(44 / 48)$ & 0.033 \\
Age 15 - 19 & $77.8(14 / 18)$ & $97.3(72 / 74)$ & 0.013 \\
Age 20 - 24 & $0.0(0 / 5)$ & $52.9(9 / 17)$ & 0.054 \\
Education-Low & $55.6(5 / 9)$ & $98.0(49 / 50)$ & 0.001 \\
Education-High & $64.3(9 / 14)$ & $78.1(32 / 41)$ & 0.313 \\
HIV Knowledge-Low & $85.7(6 / 7)$ & $93.8(30 / 32)$ & 0.457 \\
HIV Knowledge-High & $50.0(8 / 16)$ & $86.4(51 / 59)$ & 0.004 \\
\hline
\end{tabular}


more participants who had a high SD score reported abstinence than in the low SD score group $(52.9 \%$ and $0.0 \%$, respectively). However, this difference was not statistically significant (p-value 0.054 ), most likely due to the small sample size of the $20-24$ age group.

An overwhelming majority of those who had low education and who were in the high SD group reported abstinence $(98.0 \%)$ while just over half of those in the low education and low SD group reported abstinence (55.6\%). This difference was statistically significant (pvalue 0.001 ). A similar trend was observed in the high education group $(78.1 \%$ of the high SD group reported abstinence compared with $64.3 \%$ of the low SD score group), though this difference was not significant (pvalue 0.313 ).

Finally, the association between abstinence and SDS scores was examined in relation to HIV knowledge. In the low HIV knowledge group, those with high SD scores were more likely to report abstinence than those in the low SD score group (93.8\% and 85.7\%, respectively), though this difference was not statistically significant (p-value 0.457). In the high HIV knowledge group, $86.4 \%$ of the high SD score participants reported abstinence compared with the low SD score group, in which $50.0 \%$ of participants reported abstinence. This difference was statistically significant (p-value 0.004$)$.

\section{Discussion}

In the present study, we examined the relationship between social desirability and primary abstinence among Ethiopian youths who participated in an HIV intervention study. Our data showed a 13-fold difference in self-reports of primary abstinence between individuals with a high SD score compared to those with a low SD score. The findings corroborated our original hypothesis that individuals who scored high on the MC-SDS were more likely to be influenced by social norms and to over-report favorable behaviors. These individuals have a higher tendency to want to be viewed positively among their peers, and thus, would be more likely to give responses regarding sexual behaviors that would conform to societal expectations.

Several factors were assessed to determine the influence on the relationship between social desirability and self-reporting of abstinence. Our data indicated that gender does not affect the relationship between SD and self-reported abstinence. It is difficult to interpret if age is a factor that affects SD. Both age groups showed a trend that individuals in the high SD group were more likely report abstinence. However, the relationship between SD and reported abstinence among the older age group was not statistically significant. This was likely due to the small sample size of the older sub-group. The education level of the participant could be a factor that affects SD. People with higher education showed no significant difference in the reporting of abstinence. Those with low education were more affected by SD, as noted in the disparity in reports of abstinence between the high and low SD score groups. Surprisingly, the high HIV knowledge group was more affected by SD in reports of abstinence than the low HIV knowledge group. The high HIV knowledge group had a statistically significant difference in reports of abstinence between the two SD score groups, compared to the low HIV knowledge group. Perhaps with increased HIV knowledge, there is also more awareness that certain sexual behaviors that may increase the risk of contracting HIV are not condoned by society. People with higher HIV knowledge may therefore have greater susceptibility to SD and are more likely to report abstinence.

Whatever factors mediate the influence of social desirability bias on reports of abstinence, the association between SD and the self-reporting of sensitive subject matters has potentially important implications for how HIV research data in Ethiopia is interpreted, especially in research that looks at sexual behavior. From this study, it appears that SD bias is high in Ethiopia. Thus, any data collection that uses face-to-face interviews with questions that contain culturally sensitive subjects is potentially susceptible to SD and misreporting. It is unclear whether self-administered questionnaires or survey methods other than face-to-face interviews may reduce SD bias.

This study had a few notable limitations. While the tool has been validated, there may still have been problems with the MC-SDS. The instrument was initially developed for use in the United States. As such, some concepts may be tailored towards participants in highincome countries. Key concepts in the some MC-SDS statements may also have been lost during the translation. In addition, participants may have been less comfortable answering questions truthfully regarding sexual behavior when they were interviewed face-to-face. The respondents' concerns of feeling judged by research staff or lack of confidentiality may come into play. These concerns may accentuate the SD effect. Finally, since the study was conducted alongside the monitoring and evaluation of another program with budget constraints, the sample size was sub-optimal to further the understanding of how different demographic characteristics or factors may affect the relationship between social desirability and self-reports of sexual behaviors.

With these limitations, reproducing these results in Ethiopia with a larger sample size, powered to detect statistical differences in the various factors that may affect $\mathrm{SD}$ in sub-group analyses, would be a reasonable next step in research on SD in Ethiopia in regards to HIV prevention and sexual behaviors. Conducting a study to understand the relationship between SD bias and self- 
reports of sexual behaviors in other African countries would also be of value.

\section{Conclusion}

There is a relationship between high SD scores and higher reports of primary abstinence. This suggests that those who are more susceptible to social desirability bias are more likely to report abstinence. This association has potentially important implications for HIV programming at large. Many HIV programs rely on self-reported surveys to evaluate outcomes and impacts. If SD reflects the possibility that respondents misrepresent socially undesirable behavior, then the validity of self-reported survey data comes into question, and the effectiveness of HIV prevention programs may be over-estimated. It is important for investigators conducting studies by using self-reports of sensitive information to assess the potential of social desirability bias to affect the results.

\section{REFERENCES}

[1] “2011 UNAIDS World AIDS Day Report,” UNAIDS, Geneva, 2011.

[2] W. L. Kirungi, J. Musinguzi, E. Madraa, N. Mulumba, T. Callejja, P. Ghys and R. Bessinger, "Trends in Antenatal HIV Prevalence in Urban Uganda Associated with Uptake of Preventive Sexual Behaviour," Sexually Transmitted Infections, Vol. 82, Suppl. 1, 2006, pp. i36-i41. http://dx.doi.org/10.1136/sti.2005.017111

[3] A. Mahomva, S. Greby, S. Dube, O. Mugurungi, J. Hargrove, D. Rosen, K. L. Dehne, S. Gregson, M. St Louis and S. Hader, "HIV Prevalence and Trends from Data in Zimbabwe, 1997-2004,” Sexually Transmitted Infections, Vol. 82, Suppl. 1, 2006, pp. i42-i47. http://dx.doi.org/10.1136/sti.2005.019174

[4] J. A. Catania, D. R. Gibson, D. D. Chitwood and T. J. Coates, "Methodological Problems in AIDS Behavioral Research: Influences on Measurement Error and Participation Bias in Studies of Sexual Behavior," Psychological Bulletin, Vol. 108, No. 3, 1990, pp. 339-362. http://dx.doi.org/10.1037/0033-2909.108.3.339

[5] R. Tourangeau and T. Yan, "Sensitive Questions in Surveys,” Psychological Bulletin, Vol. 133, No. 5, 2007, pp. 859-883. http://dx.doi.org/10.1037/0033-2909.133.5.859

[6] M. L. Plummer, D. A. Ross, D. Wight, J. Changalucha, G. Mshana, J. Wamoyi, J. Todd, A. Anemona, F. F. Mosha, A. I. Obasi and R. J. Hayes, "A Bit More Truthful: The Validity of Adolescent Sexual BehaviourData Collected in Rural Northern Tanzania Using Five Methods," Sexually Transmitted Infections, Vol. 80, Suppl. 2, 2004, pp. ii49-ii56. http://dx.doi.org/10.1136/sti.2004.011924

[7] D. Schopper, S. Doussantousse and J. Orav, "Sexual Behaviors Relevant to HIV Transmission in a Rural African Population. How Much Can a KAP Survey Tell Us?” Social Science \& Medicine, Vol. 37, No. 3, 1993, pp. 401412. http://dx.doi.org/10.1016/0277-9536(93)90270-E

[8] C. A. Kelly, E. Soler-Hampejsek, B. S. Mensch and P. C.
Hewett, "Social Desirability Bias in Sexual Behavior Reporting: Evidence from an Interview Mode Experiment in Rural Malawi," International Perspectives on Sexual and Reproductive Health, Vol. 39, No. 1, 2013, pp. 1421. http://dx.doi.org/10.1363/3901413

[9] J. R. Glynn, N. Kayuni, E. Banda, F. Parrott, S. Floyd, M. Francis-Chizororo, M. Nkhata, C. Tanton, J. Hemmings, A. Molesworth, A. C. Crampin and N. French, "Assessing the Validity of Sexual Behaviour Reports in a Whole Population Survey in Rural Malawi,” PLoS One, Vol. 6, No. 7, 2011, p. e22840. http://dx.doi.org/10.1371/journal.pone.0022840

[10] C. J. Hopwood, C. G. Flato, S. Ambwani, B. H. Garland and L. C. Morey, "A Comparison of Latino and Anglo Socially Desirable Responding,” Journal of Clinical Psychology, Vol. 65, No. 7, 2009, pp. 769-780. http://dx.doi.org/10.1002/jclp.20584

[11] D. P. Crowne and D. Marlowe. "A New Scale of Social Desirability Independent of Psychopathology,” Journal of Clinical Psychology, Vol. 24, No. 4, 1960, pp. 349-354.

[12] T. P. Johnson and M. Fendrich, "A Validation of the Crowne-Marlowe Social Desirability Scale,” 57th Annual Meeting of the American Association for Public Opinion Research, St. Pete Beach, 2002.

[13] C. A. Latkin and D. Vlahov, "Socially Desirable Response Tendency as a Correlate of Accuracy of Self-Reported HIV Serostatus for HIV Seropositive Injection Drug Users,” Addiction, Vol. 93, No. 8, 1998, pp. 1191-1197. http://dx.doi.org/10.1046/j.1360-0443.1998.93811917.x

[14] A. W. Tatman, M. T. Swogger, K. Love and M. D. Cook, "Psychometric Properties of the Marlowe-Crowne Social Desirability Scale with Adult Male Sexual Offenders,” Sexual Abuse, Vol. 21, No. 1, 2009, pp. 21-34.

[15] A. Lange, B. Dehghani and E. de Beurs, "Validation of the Dutch Adaptation of the Buss-Durkee Hostility Inventory," Behaviour Research and Therapy, Vol. 33, No. 2, 1995, pp. 229-233. http://dx.doi.org/10.1016/0005-7967(94)00044-K

[16] D. E. Morisky, A. Angand and C. D. Sneed, "Validating the Effects of Social Desirability on Self-Reported Condom Use Behavior among Commercial Sex Workers," AIDS Education and Prevention, Vol. 14, No. 5, 2002, pp. 351-360. http://dx.doi.org/10.1521/aeap.14.6.351.24078

[17] A. Vu, N. Tran, K. Pham and S. Ahmed, "Reliability of the Marlowe-Crowne Social Desirability Scale in Ethiopia, Kenya, Mozambique, and Uganda," BMC Medical Research Methodology, Vol. 11, No. 1, 2011, p. 162. http://dx.doi.org/10.1186/1471-2288-11-162

[18] A. Edwards, "The Measurement of Personality Traits by Scales and Inventories,” Holt, Rinehart \& Winston, New York, 1970.

[19] H. Eysenck and S. Eysenck, "Manual of Eysenck Personality Inventory,” University Press, London, 1964.

[20] L. J. Cronbach, "Coefficient Alpha and the Internal Structure of Tests,” Psychometrika, Vol. 16, No. 3, 1951, pp. 297-333. http://dx.doi.org/10.1007/BF02310555

[21] Centers for Disease Control, EpiInfo, Version 3.5.4, 2012. http://wwwn.cdc.gov/epiinfo/html/prevVersion.htm

[22] StataCorp, Stata/IC, Version 11.2, 2009. 